\title{
Sobre a importância de examinar diferentes ambientes online em estudos de deliberação
}

\author{
Rousiley C. M. Maia \\ Patrícia G. C. Rossini \\ Vanessa V. de Oliveira \\ Alicianne G. de Oliveira
}

\section{I ntrodução}

Apreender deliberação, conversação informal online e a interface entre esses dois fenômenos exige que se compreendam as condições de interação e as especificidades dos diversos ambientes conversacionais da internet. O objetivo deste artigo é demonstrar a importância de analisar diferentes ambientes virtuais - no caso, os blogs, os sites de redes sociais e as plataformas colaborativas - considerando como suas especificidades técnicas podem fomentar, constranger ou impedir diferentes formas de comunicação mediada à luz da teoria deliberativa. Os três ambientes escolhidos representam os tipos de ferramentas mais populares da Web 2.0 e apresentam características específicas que permitem uma análise da utilização da internet como espaço de conversação política em esferas distintas, cada uma com constrangimentos e affordances próprios.

Adotamos a noção de sistema deliberativo (Mansbridge, 1999; Mansbridge et al., 2012; Maia, 2012; Neblo, 2005), que permite refletir sobre como a deliberação ocorre na sociedade considerando seus múltiplos ambientes e agentes. Essa abordagem valoriza a conversação cotidiana como uma importante etapa de processos mais complexos de deliberação e participação cívica (Mansbridge, 1999; Maia, 2012; Marques e Maia, 2010).

A internet representa um ambiente extremamente diverso para a interação social, cujas características transformam e reorganizam as práticas sociais. A comunicação entre indivíduos e grupos, bem como a organização de comunidades de interesse e ambientes para discussão, é característica inerente à evolução das ferramentas da internet - desde a Web 1.0, caracterizada pelos sites estáticos e pela interatividade limitada, nos anos 1990, até as plataformas da Web 2.0, cuja ênfase está 
na interação social, no compartilhamento de informações e no acesso às ferramentas de produção (Ackland, 2013; Baym, 2010). Dado seu potencial para a comunicação descentralizada, simultânea e de baixo custo, os sites de redes sociais são vistos como plataformas que dão suporte a uma série de atividades cotidianas, tais como a conversação interpessoal, o compartilhamento de conteúdos, a formação de grupos e comunidades de interesse etc. (Baym, 2010; Burgess e Green, 2009; Jenkins, 2009; Jenkins et al., 2012).

Apesar de diversos autores destacarem a diversidade de plataformas e ferramentas de comunicação virtuais, poucos estudos se dedicam a examinar essa complexidade. Redes e mídias sociais, wikis e plataformas de conteúdo colaborativo, por exemplo, alteram dramaticamente a infraestrutura da conectividade social e tornam o ambiente político mais poroso. Esse fato não é um fenômeno recente. Há décadas, estudos sobre a convergência de media mostram como as interfaces entre a arquitetura tecnológica da televisão, dos jornais, do cinema e da internet desafiam os modelos tradicionais da comunicação de massa organizada de modo centralizado, com as elites operando como gatekeepers e assegurando o monopólio da informação (Ackland, 2013; Chadwick, 2013; J enkins, 2006; J enkins et al., 2012).

Vários autores têm se concentrado em identificar os efeitos decorrentes das escolhas de design e a interface na participação política e na deliberação virtual (Coleman e Moss, 2012; Janssen e Kies, 2004; Wright e Street, 2007). Outros estudos, baseados em análise de conteúdo, estão principalmente interessados em medir regularidades e generalizações sobre discussões online, através de indicadores consagrados tais como respeito, troca de argumentos racionais, reflexividade, não coerção e inclusividade (Dahlberg, 2001; Graham, 2008; Kies, 2010; Wilhelm, 2000). Esses estudos têm oferecido ferramentas com crescente grau de sofisticação para tratar da deliberação no ambiente digital. Não obstante, essa literatura raramente interage. Daí a importância de promover um diálogo entre as contribuições desses estudos, a fim de examinar de modo integrado as condições da interação online.

Partimos da premissa que os critérios da deliberação são exigentes e que abordagens demasiadamente restritas a essa perspectiva, no âmbito da internet, podem deixar de captar fenômenos comunicativos relevantes que não configuram, necessariamente, deliberação (Bächtiger et al., 2009; Chadwick, 2009; Coleman e Moss, 2012; Dahlberg, 2004; Dahlgren, 2005; Maia, 2014). Nesse sentido, entendemos que os parâmetros normativos devem ser tomados como condições ideais para a deliberação, mesmo quando a observação da realidade não corrobora esses princípios. Ademais, metodologias desenvolvidas para apreender conversações que ocorrem em plataformas digitais, embora possam ser influenciadas pela deliberação, devem ir além dos princípios normativos para desvelar as características específicas dessas formas de comunicação. Afinal, a deliberação é um processo "raro, exigente e frágil" (Warren, 
ROUSILEY C. M. MAIA ET AL.

2007, p. 276, ver também Habermas, 1997). A argumentação, ou os momentos deliberativos, ocorre episodicamente em meio a diversas modalidades de comunicação.

Cabe destacar que examinar a conversação informal online, com a devida consideração das especificidades das diferentes formas de comunicação mediada por computador, ainda se apresenta como um desafio metodológico. Geralmente, a conversação informal é difícil de ser observada e examinada sistematicamente, uma vez que é uma atividade contínua, com múltiplos propósitos e formas de engajamento com o outro. Por isso, critérios de deliberação nem sempre são adequados e satisfatórios para a sua análise. Diante da necessidade de uma reflexão teórico-metodológica voltada para essa prática, nosso artigo examina a relação entre o design e a organização dos espaços virtuais e seus possíveis impactos na conversação mediada - refletindo, como pano de fundo, acerca das condições de deliberação.

Nosso objetivo neste artigo é caracterizar, de modo comparativo, três espaços de conversação online: blogs, sites de redes sociais (como o Facebook) e plataformas de conteúdo colaborativo (como o YouTube). A análise concentra-se nos seguintes aspectos desses ambientes: a identificação dos participantes; a moderação; as características das interações entre os usuários e a natureza do público presumido. Nossa proposta se alinha às abordagens que propõem a observação da Web 2.0 como uma ecologia complexa (Chadwick, 2013; Papacharissi, 2010; Walther et al., 2011; Maia, 2014). Nesse sentido, defendemos o argumento que uma caracterização cuidadosa das condições de comunicação de cada ambiente conversacional da internet possibilita avançar a reflexão sobre as oportunidades e os constrangimentos que essas ferramentas apresentam à interação. A análise das especificidades do ambiente online contribui para elucidar como aqueles elementos interferem na maneira através da qual as pessoas interagem online, como elas reconhecem seus interlocutores, constroem e apresentam seus argumentos e lidam com perspectivas desafiantes. Para expor essa perspectiva, não analisamos casos específicos. Em vez disso, partimos da exploração crítica da literatura corrente e estudos empíricos sobre ambientes digitais e deliberação e propomos um diálogo com evidências decorrentes de pesquisas já desenvolvidas. Essa proposta nos ajuda a reexaminar essas contribuições à luz de uma perspectiva sistêmica.

Este artigo está estruturado da seguinte maneira: na primeira seção, "Sobre ambientes conversacionais: desenhos interativos e parâmetros da deliberação", refletimos sobre as condições de comunicação mediada por computadores a partir das oportunidades e dos constrangimentos da interface, ou seja, a arquitetura dos espaços de discussão. Na seção seguinte, "Influências da interface das plataformas da internet na conversação cotidiana", examinamos de que forma as especificidades de três diferentes ambientes conversacionais - blogs, sites de redes sociais e plataformas de conteúdo colaborativo - podem fomentar ou restringir formas comunicativas e 
expressões políticas. Como conclusão, indicamos algumas implicações de nosso trabalho para pensar, metodologicamente, a conversação informal online em uma perspectiva deliberacionista.

\section{Sobre ambientes conversacionais: desenhos interativos e parâmetros da deliberação}

O uso cada vez mais frequente e ubíquo de tecnologias digitais no cotidiano suscita o questionamento sobre como as plataformas interacionais podem impactar as relações sociais por elas mediadas. A arquitetura e a organização das plataformas interferem substancialmente nas condições de participação e nas modalidades de comunicação possíveis. Esses elementos criam affordances ${ }^{1}$ - aqui traduzidas como oportunidades para ação - para as relações sociais mediadas, bem como constrangimentos e restrições a determinados tipos de comportamento e interação.

Considerando o notável efeito que as escolhas de design e a organização dos espaços de interação podem ter sobre a conversação (Davies e Chandler, 2012; Janssen e Kies, 2004; Sampaio, Barros e Morais, 2012; Wright e Street, 2007), é fundamental que as características de um determinado ambiente conversacional sejam tomadas como importantes aspectos na análise de fenômenos comunicativos que ocorrem no ambiente virtual.

Nesse sentido, Baym (2010) enumera sete conceitos-chave para refletir acerca de como o uso de tecnologias digitais (especificamente internet e celulares) modifica as interações e, potencialmente, os relacionamentos mediados. São eles: a) a interatividade - social (conexões entre pessoas e grupos) ou técnica (interação com a tecnologia e suas plataformas); b) a estrutura temporal; c) as "pistas" sociais informações sobre contexto, conteúdos, significados e identidades; d) o alcance; e) a replicabilidade; f) o arquivamento; e g) a mobilidade (Baym, 2010, p. 6-12). Cabe ressaltar que essas características variam em diferentes ambientes conversacionais e, portanto, devem ser observadas a priori como condições das interações mediadas.

A estrutura temporal da comunicação é um aspecto técnico que estabelece o padrão de interação. Via de regra, as conversações virtuais podem ser síncronas ou assíncronas. O primeiro tipo requer a presença dos interlocutores num mesmo ambiente e em tempo real, sendo uma modalidade mais próxima das interações face a face. São exemplos os chats e as ferramentas de mensagens instantâneas. Já a comunicação

\footnotetext{
1 O termo affordances, originalmente cunhado por James Gibson em 1977, refere-se ao campo da ecologia. Ele sugere que humanos e animais são agentes híbridos, em certo sentido, porque eles dependem fortemente do ambiente e daquilo que ele oferece. Assim, a noção de Gibson provê um quadro de referências geral sobre a maneira como organismos percebem objetos e são estimulados a agir a partir das qualidades que são capazes de perceber no mundo. Assim, affordances seriam as oportunidades para ação que um ser vivo é capaz de perceber no ambiente.
} 
ROUSILEY C. M. MAIA ET AL.

assíncrona refere-se a modalidades de comunicação na qual os interlocutores podem participar em momentos distintos, tais como e-mails, listas e grupos de discussão, fóruns, comentários em blogs etc.

A observação sobre a estrutura temporal das interações possui desdobramentos relevantes sobre os graus de controle que interlocutores detêm sobre a própria expressão e o engajamento com o outro (Boyd, 2007, 2011; Donath e Boyd, 2004). Para Baym (2010, p. 4), "a separação entre presença e comunicação nos oferece mais controle sobre nossos mundos sociais, mas, ao mesmo tempo, nos submete a novos mecanismos de controle, vigilância e constrangimento". A autora destaca que os ambientes de conversação síncrona aumentam a sensação de proximidade entre os interagentes, mesmo quando estão fisicamente distantes. O sincronismo produz uma constante negociação do relacionamento entre os participantes e o público, o que torna as mensagens mais imediatas e pessoais e encoraja comportamentos descontraídos (Baym, 2010, p. 8). O custo da interação síncrona é que, normalmente, o número de participantes é mais restrito porque essa modalidade requer que as pessoas estejam conectadas ao mesmo tempo.

Já nas situações em que indivíduos interagem por meios assíncronos, é possível mobilizar grupos significativamente maiores e os participantes têm mais tempo para construir e manter estrategicamente suas presenças online. Como Janssen e Kies (2004) apontam, enquanto conversas em tempo real tendem a ser mais informais, a interação assíncrona favorece debates mais racionais ou críticos e proporcionam aos interagentes mais tempo e espaço para apresentar e desenvolver seus argumentos.

Uma característica que distingue a conversação informal mediada é a transversalidade (transversability) (Brundidge, 2009), ou seja, a capacidade que os indivíduos têm de realizar diversas atividades simultaneamente. Um internauta pode, por exemplo, acessar uma notícia em uma página da internet, compartilhá-la em sua rede social, comentá-la em modo privado por meio de mensagens instantâneas ou engajar-se em debates nas seções de comentários ou em fóruns específicos. A ecologia complexa dos media digitais requer a consideração de que ambientes conversacionais distintos apresentam características que os tornam mais suscetíveis ao surgimento de certos modos de comunicação - o que não significa, em última instância, que as pessoas que se comportam de certa maneira em um ambiente de discussão não agem de forma diferente quando as condições de interação são alteradas. Em outras palavras, ainda que certos ambientes fomentem interações caracterizadas por civilidade e respeito, os participantes desse espaço podem estar, simultaneamente, debatendo o tema em espaços privados ou redes que suscitam comentários intolerantes ou radicais.

Na próxima seção, "Influências da interface das plataformas da internet na conversação cotidiana", apresentamos uma proposta analítica das características que podem afetar o engajamento discursivo, a partir dos exemplos de três ambientes 
virtuais: blogs, sites de redes sociais e plataformas colaborativas. Tomando como base os critérios identificados na literatura de deliberação, refletimos sobre o modo pelo qual os constrangimentos e as oportunidades dos variados ambientes conversacionais da internet impactam a configuração da argumentação e da conversação mediada. Nesse sentido, mais do que pensar em plataformas específicas - a rede social ou a plataforma "mais atual" - , é preciso contextualizar as características que identificam tais meios, de modo que a análise permaneça relevante ainda que determinadas plataformas se tornem obsoletas.

Para empreender esse esforço, é preciso, primeiramente, pontuar brevemente a operacionalização dos critérios deliberativos na literatura. Apesar das diferentes correntes teóricas que se dedicam a refletir sobre a democracia deliberativa, há um relativo consenso de que a deliberação pública é o processo através do qual os cidadãos, em condições de liberdade e igualdade, mobilizam e trocam argumentos com justificações compreensíveis e passíveis de serem aceitas pelos demais, a fim de tomar decisões legítimas e democráticas. Apesar de inúmeras controvérsias normativas sobre os critérios em questão, a deliberação pode ser entendida, de modo sintético, como uma prática de discussão refletida, em que os parceiros se engajam de modo recíproco e responsivo com os argumentos e as justificações dos outros (Benhabib, 1996; Bohman, 1996; Cohen, 1997, Dryzek, 2000; Gutmann e Thompson, 1996, 2004; Habermas, 1997; Maia, 2012; Steiner, 2012; Steiner et al., 2004).

Para os propósitos da reflexão em tela, tomamos como definição os apontamentos sintéticos dos indicadores de deliberação adaptados para o ambiente online. Para teóricos que compartilham da matriz habermasiana, tais como Dahlberg (2004) e Kies (2010), a deliberação precisa atender a cinco critérios normativos: justificação racional, cujo objetivo é verificar se as posições apresentadas são debatidas criticamente e os argumentos são fundamentados; reflexividade, referente à disposição dos interagentes em avaliar a posição dos outros e rever seu posicionamento inicial; empatia ou ideal role taking, que considera o respeito e a tolerância às colocações de outros indivíduos; sinceridade, que, embora seja difícil de aferir discursivamente, está relacionada à consistência e coerência do discurso; inclusão, concernente à possibilidade de todo interessado ou afetado por um determinado tópico ser capaz de participar da discussão; igualdade discursiva, dizendo respeito à igual possibilidade de introduzir e questionar qualquer proferimento relativo às normas, necessidades, 
atitudes etc. (Dahlberg, 2004, p. 32-35; Kies, 2010, p. 42) ${ }^{2}$.

Ao recorrer aos critérios deliberativos para observar trocas comunicativas informais, é importante reiterar que a deliberação é um fenômeno raro, com princípios normativos exigentes e de difícil observação empírica. De tal modo, não pretendemos defender a tese de que as trocas argumentativas que acontecem em espaços virtuais possam cumprir todos os requisitos da deliberação. Não obstante, identificar as relações que se estabelecem entre as modalidades das conversações políticas que acontecem em ambientes digitais - a partir dos critérios de deliberação - é útil para refletir sobre a maneira pela qual diferentes configurações interativas facilitam ou dificultam práticas deliberativas.

\section{I nfluências da interface das plataformas da internet na conversação cotidiana}

Pesquisadores que têm na internet seu objeto de estudo não podem deixar de considerar que as ferramentas digitais são, fundamentalmente, ambientes construídos. As escolhas de design e organização - considerando-se aspectos de desenvolvimento, programação e administração, que precedem o desenvolvimento da interface - definem e delimitam as condições e modalidades através das quais usuários poderão interagir tanto com a mídia quanto entre si. A visão da internet como um ambiente construído, para Coleman e Moss (2012), é o ponto de partida para a compreensão das limitações e possibilidades de ação e participação política em uma dada plataforma. Esses autores recomendam, portanto, um exame das decisões estratégicas que determinam as maneiras possíveis de interação, mobilização e ação social online.

Com a preocupação de investigar especificamente ferramentas de participação política e engajamento cívico, Janssen e Kies (2004) identificam quatro fatores que podem afetar a discussão: identificação; abertura e liberdade discursiva; moderação; agenda setting. A literatura focada em conversação mediada por computador e sites de redes sociais, cujo interesse recai sobre a construção de identidades e comportamento em ambientes virtuais, destaca que aspectos como a identificação dos participantes, a liberdade discursiva e a moderação são elementos que influenciam o engajamento discursivo e a interação social mediada. Esses aspectos contribuem para moldar a autoexpressão, a modalidade de negociação entre igualdades e diferenças entre os

\footnotetext{
2 Dahlberg (2004) aponta, ainda, como sexto critério, "autonomia em relação ao Estado e ao poder econômico", critério que pode ser avaliado em relação à liberdade de expressão diante de forças coercivas diretas, como censura e vigilância, e de conteúdos estratégicos. Kies (2010) lista também "pluralidade", dizendo respeito à pluralidade de vozes no contexto deliberativo, e, ainda, "impacto externo", referindo-se ao impacto nas opiniões formadas e decisões tomadas, fora do contexto do debate. Para nossos propósitos, essas dimensões não são consideradas, uma vez que não se encontram entre aqueles parâmetros mais consensuais na literatura sobre deliberação.
} 
participantes e, ainda, a construção de comunidades (Baym, 2010; Boyd, 2007, 2011; Boyd e Ellison, 2007; Donath e Boyd, 2004; Ellison e Boyd, 2013).

Por identificação, entende-se a autoapresentação do indivíduo na internet. Nas plataformas digitais, isso se traduz na necessidade de registro e/ou uso de perfis virtuais em determinada plataforma, o que afeta substancialmente a participação e o comportamento dos usuários por diferentes motivos. Trata-se de uma questão controversa: por um lado, o anonimato contribui para aumentar a liberdade de expressão dos sujeitos, sobretudo em debates sobre temas constrangedores ou tabus. Por outro lado, o anonimato, ao resguardar a identidade dos interagentes, pode facilitar a emergência de comportamentos agressivos, desrespeitosos e preconceituosos.

Davies e Chandler (2012) observam que o anonimato pode aumentar a disposição dos indivíduos em participar do debate, mas tende a reduzir o sentimento de satisfação destes devido à distância emocional entre interlocutores - ou seja, em ambientes onde a identidade real é resguardada pela utilização de apelidos ou onde não há necessidade de apresentar alguma forma de identificação para participar. Ademais, os indivíduos tendem a tratar seus interlocutores com distanciamento e a não considerar suas opiniões, o que, por fim, contribui para que as trocas discursivas apresentem níveis mais baixos de respeito e reflexividade.

O uso de identidades reais, por outro lado, pode gerar constrangimentos e restrições à liberdade de expressão ao vincular as ações virtuais a uma pessoa que pode ser facilmente identificada. Não obstante, esse pode ser um aspecto positivo nos ambientes de interação social porque a possibilidade de rastreamento da identidade tende a reduzir comportamentos indesejáveis, tais como o uso de mentiras e argumentos falsos, a violência e o desrespeito nas discussões em rede (Boyd e Ellison, 2007; Donath e Boyd, 2004; Janssen e Kies, 2004, Maia e Rezende, 2014a).

Para Baym (2010, p. 31-32), a incerteza inerente às construções identitárias na internet é uma característica marcante dos ambientes discursivos nos quais indivíduos interagem em condições de anonimato. Segundo a autora, a ausência de contexto (ou de "pistas" sociais) contribui para que os indivíduos tenham dificuldade em acreditar que os seus parceiros de interação correspondam às suas identidades construídas virtualmente.

O critério abertura e liberdade discursiva refere-se à possibilidade de que o acesso ou o número de interações de cada usuário seja limitado, o que, sob uma perspectiva deliberacionista, afetaria a pluralidade de ideias e discussões no curso de um debate (Janssen e Kies, 2004; Kies, 2010). Esse critério, relacionado à ideia da igualdade discursiva na deliberação, é bastante complexo e de difícil operacionalização em pesquisas sobre deliberação virtual (Dahlberg, 2004, 2007; Sampaio, Barros e Morais, 2012). É também uma questão controversa. Isso porque a internet, por um lado, cria obstáculos de naturezas distintas à participação dos cidadãos, o que traz 
ROUSILEY C. M. MAIA ET AL.

novos desafios e problemas de desigualdades para a inclusão democrática (Norris, 2000; Papacharissi, 2004). Por outra leitura, é possível defender o argumento de que ambientes digitais são democráticos e inclusivos na medida em que garantem condições mais igualitárias para aqueles que possuem meios e motivações para participar (Rossini e Maia, 2014).

A presença de moderação em espaços conversacionais pode exercer dois papéis: a) o de censurar perspectivas que não são congruentes com as normas e posições políticas propostas para o espaço - o que seria negativo do ponto de vista da pluralidade e da liberdade de expressão - e b) o de promover a deliberação, oferecendo oportunidades para a expressão de opiniões minoritárias, sintetizando o debate, provendo informações adicionais sobre o assunto etc.

Finalmente, por agenda setting os autores se referem ao controle sobre a determinação do tópico: os participantes (agenda descentralizada), os organizadores (agenda centralizada) ou ambos (agenda parcialmente centralizada). Em outras palavras, esse critério está interessado em observar em que nível está o controle sobre o tema das discussões virtuais - se é definido a priori, negociado entre os participantes e os administradores, ou se os interagentes possuem liberdade para propor novos debates (Janssen e Kies, 2004, p. 5). Evidentemente, esse critério focaliza ambientes formais de participação política e ressalta o papel delegado ao cidadão nesses espaços (Chadwick, 2013).

A seguir, considerando as condições de interação de ambientes conversacionais distintos - os blogs, sites de redes sociais e plataformas colaborativas -, buscamos apreender formas comunicativas e expressões políticas que não se caracterizam como deliberação, mas como interações sociais menos pretensiosas, nas quais a relação é negociada a todo momento entre interlocutores que falam a uma audiência imaginada (Chadwick, 2003; Coleman e Blumler, 2009; Coleman e Moss, 2012; Boyd, 2011).

Blogs

Os blogs surgiram na década de 1990 como espaços em que indivíduos escreviam sobre questões de caráter pessoal ou público (Ackland, 2013; Baym, 2010). Embora a interface e os recursos disponíveis aos usuários de blogs tenham naturalmente evoluído na medida em que as plataformas da internet se tornaram mais interativas, a arquitetura técnica e a organização desses espaços apresentam linhas gerais mais ou menos bem definidas, que transcendem a multiplicidade de plataformas que oferecem o serviço. Também é importante ressaltar que os blogs não configuram, necessariamente, ambientes conversacionais. No entanto, a interação social é possível por meio da seção de comentários - que pode ser moderada pelo proprietário do blog.

Os blogs apresentam propostas bem definidas, que ficam a critério de seu 
"criador": podem ser um espaço de reflexão pessoal ou de comentários sobre acontecimentos, ou um ambiente especializado (como blogs jornalísticos, de colunistas e figuras públicas etc.). Podem tratar de questões de interesse público, como política e economia, ou versar sobre entretenimento, moda, games etc. A natureza temática do blog delimita sua audiência imaginada - o público para o qual o autor escreve e ao qual se dirige - e fomenta relações entre blogueiros com interesses afins. Nesse âmbito, a definição de agenda é predominantemente centralizada e acontece de cima para baixo, ou seja, do autor para o público.

Muitas vezes, blogueiros criam relações com autores de outros blogs e disponibilizam links para seus sites no blogroll, que também inclui links unilaterais para blogs e sites que o autor considera interessantes (Baym, 2010). A interação social em blogs é assíncrona e ocorre, predominantemente, na seção de comentários - espaço no qual o autor do blog pode interagir com sua audiência presumida.

No entanto, esse relacionamento é sempre desigual porque o blogueiro possui controle sobre os comentários e pode decidir quais opiniões ficarão visíveis no blog e quais serão apagadas. Aqui, cabe analisar a necessidade ou não de identificação do usuário - definição que também fica a critério do autor - e a presença de moderação, fatores que impõem constrangimentos à participação desrespeitosa e ao uso de identidades falsas (Boyd, 2007, 2011; Donath e Boyd, 2004; Maia e Rezende, 2014a, 2014b). Essas características de design e organização do blog, cuja influência reside na natureza comunicativa do espaço, podem fomentar ou restringir a emergência de determinadas características deliberativas. No entanto, é importante ressaltar que blogs são ferramentas amplamente customizáveis e, portanto, difíceis de apreender a partir de critérios predefinidos. As características estruturais dos blogs dependem exclusivamente das decisões de seu proprietário/criador. Mas, como ressalta Siles (2012), as identidades de usuários e das tecnologias são construídas mutuamente e isso influencia nas formas comunicativas que se realizam nesses espaços.

A estrutura da ferramenta - postagens seguidas de comentários - e a relação entre autor e leitores - que também pode ser aferida a partir da forma como os leitores são convidados a participar - tornam esse ambiente propício à troca de argumentos justificados e à presença de respeito entre os interlocutores. Isso porque: a) participações desrespeitosas podem ser censuradas; b) blogs reúnem leitores assíduos que, ao longo do tempo, podem estabelecer relações sociais tanto com o autor como com os demais leitores.

Estudos em deliberação online demonstram que blogs são ambientes profícuos para a conversação e a argumentação (Kaye, 2011; Meraz, 2007; Amaral, Recuero e Montardo, 2009). Contudo, é preciso ter em mente que a variedade de temas e objetivos dos blogs faz com que a "audiência" para a qual os autores se expressam (Kaye, 2011, p. 210) também tenha perfis variados. Afinal, a unidade e a identidade 
dos blogs também interferem na definição da identidade dos usuários como grupo, tendo em vista o compartilhamento de valores e práticas. A tese de Siles (2012) é a de que as identidades de usuários são construídas mutuamente em relação às características do blog; e isso traz implicações não apenas para o modo de engajamento das pessoas nas conversações travadas nesses espaços, mas, também, para os níveis de justificação dos comentários e, ainda, para a atitude responsiva dos participantes.

Em ambientes em que há respeito e heterogeneidade de opiniões e posições políticas, estudos apontam que o engajamento discursivo parece conduzir a argumentos mais sofisticados, o que caracterizaria um nível mais elevado de justificação (Brundidge e Rice, 2009; Risse, 2000, Steiner et al., 2004, Steiner, 2012). A troca de mensagens e a contestação de opiniões conflitantes nos blogs parece exercer pressão para que os participantes expliquem suas opiniões de modo mais claro, com maior grau de profundidade e com evidências mais convincentes, para que sejam entendidas ou aceitas pelos demais (Maia e Rezende, 2014a, 2014b). Nesse sentido, a "pressão" para que se ofereçam argumentos mais sofisticados parece favorecer a reciprocidade entre os participantes. Esse comportamento responsivo pode ocorrer, inclusive, em situações em que não há um nível considerável de reflexividade (Mutz, 2006).

A existência ou não de pluralidade de opiniões e discursos, segundo Wojcieszak e Mutz (2009), afeta substancialmente a percepção do potencial deliberativo e do valor das conversações políticas na internet. Nos estudos cujo objeto são espaços online definidos, a priori, como politicamente heterogêneos, a internet é retratada como espaço propício às trocas deliberativas entre participantes de mente aberta, que apreciam perspectivas diferentes. Do contrário, quando pesquisadores estudam grupos ligados a partidos ou ideologias, é comum que a conclusão indique a presença de "câmaras de eco", nas quais pessoas que nutrem perspectivas semelhantes são expostas a argumentos parciais, que reforçam suas predileções iniciais (Wojcieszak e Mutz, 2009, p. 42).

\section{Sites de redes sociais}

Redes sociais são ambientes complexos cuja estrutura básica é composta por perfis públicos ou semipúblicos, articulação de uma lista de conexões recíprocas e possibilidade de navegar por essas listas e visualizar tanto conexões pessoais como aquelas mantidas por outros usuários do sistema (Boyd e Elisson, 2007). Nesses ambientes, as atitudes dos usuários tendem a ser influenciadas por motivações sociais, como a vontade de participar e compartilhar conteúdos úteis ao grupo, além de construir e manter identidades públicas desejáveis para um público imaginado de pessoas com quem desejam se comunicar ou para quem desejam aparecer (Boyd, 2011; Boyd e Ellison, 2007; Donath e Boyd, 2004; Ellison e Boyd, 2013; Walther et al., 
2008).

Inicialmente, sites de redes sociais eram plataformas centradas na construção e na visualização de perfis pessoais - uma herança dos sites de relacionamentos amorosos (Ellison e Boyd, 2013) - e na articulação das listas de "amizades", relações recíprocas estabelecidas publicamente. Sob o argumento de que a saliência dessas características foi alterada pela evolução das plataformas e de seus usos, Ellison e Boyd revisaram, recentemente, a definição para os sites de redes sociais enfatizando os processos de produção e circulação de conteúdos ${ }^{3}$ :

Uma rede social é uma plataforma de comunicação interconectada na qual os participantes: 1) possuem perfis únicos e identificáveis que consistem de conteúdos criados pelo usuário, conteúdos providos por outros usuários e/ou dados em nível de sistema; 2) podem articular conexões publicamente que podem ser vistas e examinadas pelos outros; e 3) podem consumir, produzir e/ou interagir com streams de conteúdo gerado por usuários provido por suas conexões no site (Ellison e Boyd, 2013, p. 158).

No âmbito da construção de perfis - representação virtual do indivíduo -, as autoras destacam o processo colaborativo de construção da identidade entre o usuário e suas conexões: além das informações pessoais dos usuários, os comentários e fotos compartilhados por seus amigos contribuem para moldar a forma como os perfis são vistos na rede e essas informações tendem a ser provas mais confiáveis sobre a identidade do indivíduo do que aquilo que ele provê (Donath e Boyd, 2004; Ellison e Boyd, 2013; Walther et al., 2008). Considerando-se que sites de redes sociais são espaços cujas conexões sociais são predominantemente estabelecidas entre pessoas que já possuem algum tipo de relacionamento offline, a visibilidade dessas conexões interpessoais gera numerosos constrangimentos ao comportamento dos usuários (Boyd, 2007, 2011; Boyd e Ellison, 2007; Ellison e Boyd, 2013). Além disso, saber que alguém está conectado a outra pessoa já conhecida favorece o estabelecimento de confiança para novos relacionamentos, de modo que "conexões em comum" atuam como importantes "pistas" sociais, provendo contexto às interações em redes sociais (Baym, 2010; Donath e Boyd, 2004; Walther et al., 2008).

Ter a identidade revelada e estar conectado a diferentes grupos de relações sociais (família, amigos, relações de trabalho etc.) são constrangimentos sociais que

\footnotetext{
${ }^{3}$ Uma importante mudança enfatizada neste conceito é a relevância dos streams de conteúdo, tais como o feedback de notícias do Facebook, o qual se concentra mais no compartilhamento de mídia e nas atualizações de status do que nos perfis. O agregado de mídia provido pelas conexões é o ponto de partida para as demais atividades na rede social e, em geral, sua visibilidade é definida por algoritmos que mensuram a frequência das interações para identificar conteúdos relevantes de acordo com os rastros do usuário.
} 
determinam a forma através da qual os indivíduos se comportam e interagem online. No Facebook, especificamente, é possível se comunicar tanto de maneira assíncrona, por meio de posts e comentários, como de modo privado e em tempo real, utilizando o chat e as mensagens privadas (Ellison e Boyd, 2013). Dessa forma, é possível assumir que indivíduos irão se comportar de acordo com as normas percebidas de comportamento em diferentes ambientes: enquanto páginas e grupos fomentam a criação de comunidades de pessoas com interesses ou pensamentos semelhantes e podem, portanto, ser propícias a debates autorreferenciados e até hostis em relação a posições divergentes (Maia e Rezende, 2014a; 2014b), a interação das pessoas com seu círculo de conexões em espaços públicos da rede tende a se caracterizar pela tolerância e abertura aos conteúdos com posições conflitantes (Rossini, 2013) ${ }^{4}$.

No tocante aos critérios acima mencionados, a identificação é o único parâmetro estável nos diferentes ambientes comunicacionais do Facebook: para participar de qualquer atividade na rede, o usuário precisa possuir um perfil conectado a uma conta verificada de e-mail e pode ser penalizado por atitudes que violem os termos de acesso do site.

Em relação aos outros parâmetros, o Facebook apresenta condições distintas de comunicação. A definição de agenda pode ocorrer das três formas: em grupos, eventos e páginas. O tema costuma ser definido pela pessoa ou grupo de pessoas responsável pela criação e administração. Elas também possuem, nesse contexto, o controle sobre ferramentas de moderação - podendo apagar comentários e conteúdos - e podem, portanto, restringir a abertura e a liberdade discursiva. O estudo de Maia e Rezende (2014b) mostra que participantes em páginas e eventos de apoio a determinadas causas tendem a excluir ou rechaçar pessoas que contrariam as crenças dominantes no grupo. Já no âmbito dos comentários em publicações visíveis no feed de notícias, os usuários não têm o controle sobre a participação de suas conexões - podendo, portanto, passar por constrangimentos ou, ainda, ser interpelados por discussões mais heterogêneas (Rossini, 2013). Aqui, nota-se maior abertura e liberdade discursiva e a possibilidade de moderação sobre a ação de terceiros é limitada ${ }^{5}$.

A constatação de tais características do ambiente dos sites de redes sociais demonstra que esse é um espaço de forte caráter discursivo e interativo. Os usuários

\footnotetext{
${ }^{4}$ A ênfase na produção de conteúdos fez com que o Facebook modificasse o padrão de suas conexões, que, inicialmente, eram obrigatoriamente recíprocas. No momento de tessitura deste artigo, também era possível "seguir" pessoas, uma conexão unilateral que significa receber suas atualizações de status sem estar conectado como amigo. É importante enfatizar que as mudanças nas ferramentas das redes sociais ocorrem em ritmo acelerado e frequentemente sem aviso prévio, de modo que características observadas por uma pesquisa podem já estar ultrapassadas ou não existir no momento da sua publicação (Ackland, 2013; Ellison e Boyd, 2013).

${ }^{5}$ Um usuário pode definir quem, entre suas conexões, possui permissão para publicar em seu perfil e também pode ocultar atividades dele. No entanto, no âmbito das publicações em modo público, é impossível controlar tanto o conteúdo como a visibilidade.
} 
desse ambiente possuem o controle sobre a própria participação e uma autoidentificação pautada principalmente por motivações pessoais. Essa especificidade do Facebook possibilita que as conexões dos participantes com os outros usuários, que pertencem à sua rede de relacionamentos, sejam mais fortes e duradouras. Isso tende a estimular a autoexpressão e a afirmação da identidade. Diversos autores sustentam o argumento segundo o qual esses fatores facilitam a interação nos sites de redes sociais, o que abre espaço para possíveis trocas argumentativas (Ellison, Steinfield e Lampe, 2011; Grasmuck, Martin e Zhao, 2009; Valenzuela, Park e Kee, 2009; Ward, 2012). Nesse sentido, critérios de deliberação podem ser utilizados para apreender as condições de comunicação entre pessoas que se conhecem e que convivem em determinados domínios sociais.

A possibilidade de os sites de redes sociais se tornarem espaços de interação é destacada em pesquisas que investigam a formação de capital social em sites como o Facebook. De acordo com a pesquisa desenvolvida por Ellison, Steinfield e Lampe (2011), o uso do Facebook por estudantes universitários demonstra as seguintes características: a) o site torna possível que os indivíduos mantenham um amplo conjunto de laços sociais fracos; b) permite que indivíduos tornem relações efêmeras em conexões persistentes; c) diminui os custos de manter laços sociais fracos; d) pode reduzir as barreiras para iniciar interações e facilitar a formação de um terreno comum para o diálogo; e) pode tornar mais fácil a busca por informação e apoio no outro. Os pesquisadores concluem que o Facebook pode aumentar o capital social, o que, por sua vez, potencializa a possibilidade de os sites de redes sociais se tornarem arenas discursivas. Grasmuck, Martin e Zhao (2009), por exemplo, ao pesquisar as expressões de identidade no Facebook de determinados grupos étnico-raciais, demonstram que os sujeitos recorrem a diferentes estratégias, desde elementos visuais até técnicas narrativas, para estabelecer relações entre suas identidades virtuais e reais, enfatizando, sobretudo, expressões de orgulho e pertencimento étnico ou racial. Sendo assim, os sites de redes sociais podem funcionar como espaços de reforço de identidades e de pertencimento a grupos, bem como arena de disputa de interesses, com "declarações fortemente fundamentadas na vida offline" (Grasmuck, Martin e Zhao, 2009, p. 177).

No Facebook, as condições para examinar o critério de não coerção, importante para o processo deliberativo, apresenta certas especificidades. Primeiro, esse é um ambiente em que constrangimentos próprios da vida social, normas e expectativas relativas aos padrões de comportamento no círculo de pessoas conhecidas se fazem presentes com maior intensidade. Poderíamos aludir aqui aos "constrangimentos internos" (Habermas, 1997, p. 305-306), que bloqueiam a livre expressão da opinião e de discordâncias ou a inibição relacionada à autocompreensão que as pessoas nutrem sobre si e seus papéis dentro das hierarquias sociais, aos custos de conflitos explícitos 
ROUSILEY C. M. MAIA ET AL.

nas relações etc. Segundo, a automoderação em sites como Facebook pode ser elevada. Em ambientes em que as pessoas se agregam espontaneamente devido à identificação com uma causa comum, em situações de ativismo, por exemplo, é recorrente a prática entre os participantes de convidar pessoas com posicionamentos opostos a se retirarem das páginas e perfis específicos (Maia e Rezende, 2014a, 2014b). Nesse sentido, as redes sociais podem ser utilizadas tanto para nutrir solidariedade entre pessoas que se identificam, como parte de um esforço comum para fazer avançar certas causas, quanto para se diferenciar e, em alguns casos, rechaçar aqueles identificados como diferentes (Ward, 2012, p. 161).

Por fim, ressaltamos as condições de responsividade e reflexividade da deliberação. Os sites de redes sociais, como o Facebook, são ambientes mais complexos para a operacionalização do critério de reflexividade, uma vez que possibilitam múltiplas formas de comunicação que variam entre meios síncronos e assíncronos, públicos (comentários no feed de notícias), semipúblicos (grupos, eventos, páginas) e privados. No âmbito das relações sociais e interações públicas de um usuário, é plausível supor que suas interações serão mais reflexivas e respeitosas. O mesmo não ocorre quando se consideram páginas e grupos, nos quais pessoas com interesses em comum e opiniões semelhantes tendem a desrespeitar a expressão de perspectivas desafiantes. Trata-se, portanto, de uma questão que depende fundamentalmente do contexto das interações que se pretende observar.

\section{Plataformas de conteúdo colaborativo}

As plataformas de conteúdo colaborativo, frequentemente abordadas sob o conceito guarda-chuva "mídias sociais", são sites da internet que garantem aos usuários acesso gratuito e simplificado a ferramentas de produção e compartilhamento de conteúdos. São exemplos o Soundcloud, site destinado à produção de áudio, o Flickr, cujo uso é para compartilhamento de imagens e fotografias, o YouTube e o Vimeo, plataformas de vídeos, entre outras numerosas possibilidades (Ackland, 2013; Ellison e Boyd, 2013; O'Reilly, 2005; Shirky, 2011).

Assim como os sites de redes sociais, diferentes plataformas possuem ferramentas específicas para interação social. Para os objetivos deste artigo, elegemos - YouTube como exemplo para nossa reflexão acerca dos critérios de organização e design e seus possíveis efeitos sobre a interação social.

De maneira geral, a estrutura temporal da interação nesses ambientes é assíncrona e ocorre predominantemente em seções destinadas a comentários - embora seja possível acrescentar comentários e anotações no próprio vídeo. Uma diferença marcante em relação a sites de redes sociais é o fato de que os conteúdos são acessíveis àqueles que não possuem registro e, frequentemente, a participação por 
meio de comentários também é aberta ${ }^{6}$ ao público - o que significa, em termos práticos, que não é necessário estar identificado para participar de discussões nessas plataformas. A ausência de mecanismos de identificação - ou, em outras palavras, a possibilidade de anonimato - pode implicar comportamentos abusivos, falta de respeito e uso de linguagem imprópria (ver Maia e Rezende, 2014a, 2014b; Mutz, 2006; Wojcieszak e Mutz, 2009).

A seção de comentários do YouTube tem a opção de resposta - o que propicia o surgimento de discussões entre usuários - e apresenta duas formas de interação reativa - ou interação com a mídia: apoiar o comentário (a figura da mão com o dedo polegar para cima) ou negá-lo (a figura da mão com o dedo polegar para baixo). Outra forma de interação é a inserção de anotações no vídeo (caixas de texto que aparecem durante a reprodução do vídeo).

Em relação a liberdade e abertura discursiva, as plataformas de conteúdo colaborativo podem ser consideradas propícias à liberdade de expressão, uma vez que permitem a participação de indivíduos não registrados. Nesse sentido, elas são mais abertas que ferramentas que restringem a interação ao registro. No entanto, o proprietário do canal pode optar por não abrir a opção de comentários em seu conteúdo ou controlar a visibilidade dos comentários vinculando-os a uma aprovação. No tocante à definição de agenda, é possível supor que comentários feitos em um vídeo serão referentes ao conteúdo deste, de modo que a agenda da discussão seria, a princípio, determinada pelo tema da mídia. No entanto, como é possível desenvolver discussões a partir de respostas a comentários, o YouTube frequentemente se torna palco de debates entre indivíduos com opiniões divergentes sobre questões que transcendem o tema original (Maia e Rezende, 2014a).

Ao analisar a constituição e os mecanismos de participação e coerência presentes nos diálogos ambientados nos comentários do YouTube, Bou-Franch, Lorenzo-Dus e Blitvich (2012, p. 516) ressaltam que esse ambiente não é habitado somente por produtores de vídeo e espectadores, mas também por um importante grupo de usuários dedicados à interação textual. Os autores ressaltam que as interações que lá acontecem são "complexas, flexíveis, instáveis e imprevisíveis" e que as pessoas que se expressam no YouTube fazem isso principalmente de forma anônima, conscientes de que estão "interagindo e entrando em contato com outros, muitas vezes desconhecidos, YouTubers" (Bou-Franch, Lorenzo-Dus e Blitvich, 2012, p. 502). Conforme discutimos, o anonimato tende a ampliar o distanciamento entre os interlocutores e dificultar o reconhecimento destes como parceiros de interação. Naturalmente, esse fator contribui para que as trocas discursivas no YouTube

\footnotetext{
6 Usuários cadastrados podem optar por compartilhar seus conteúdos em modos privados, estando acessíveis apenas mediante senhas ou links específicos. No entanto, essa não é a prática majoritária nesses ambientes. O objetivo do compartilhamento de conteúdos costuma ser ampliar o alcance destes.
} 
apresentem menos respeito e reciprocidade, uma vez que as pessoas se encontram virtualmente na presença de uma "'massa' imaginada de usuários comuns" (Burgess e Green, 2009, p. 8), formada por usuários ativos e passivos, que acessam o site para consumir conteúdos e observar eventuais discussões.

A moderação - por onde as participações desrespeitosas poderiam ser censuradas - não é característica marcante desse ambiente, embora os administradores dos canais possam utilizar esse tipo de ferramenta. Tratamentos desrespeitosos fazem com que a audiência seja vista como uma massa de oponentes (J uris, 2005). Isso, além de não favorecer a cooperação argumentativa, propicia que, no plano da justificação, aconteça uma "guerra de interpretações" (Juris, 2005, p. 416). Em espaços como o YouTube, os participantes tendem a ser indiferentes quanto à possibilidade de os demais usuários responderem ou não às suas ideias e razões, e, ainda, desinteressados nas respostas que eles possam receber, desfavorecendo, portanto, a emergência de interações dialógicas.

Cenário diferente do que pode acontecer quando, por exemplo, não estão em jogo questões controversas. Ao analisar os comentários de vídeos do estilo "como eu faço..." veiculados no YouTube, Lindgren (2012) mostra que, apesar do relativo anonimato, há um clima participativo, em que a maioria dos comentários expressa posições moderadas - negativas ou positivas. Segundo o autor, os comentários fazem parte de um circuito midiático na plataforma, o que ajudaria, inclusive, na criação de novas conexões e relações entre os participantes. Desse modo, os comentários mais extremos e polêmicos seriam uma pequena minoria.

\section{Considerações finais}

O esforço empreendido neste artigo foi o de refletir sobre a maneira pela qual os diversos ambientes comunicacionais presentes na internet propiciam formas distintas de conversação informal. Partindo de uma observação de pesquisas sobre a interface interativa de três plataformas distintas - blogs, sites de redes sociais e sites de conteúdo colaborativo -, buscamos ressaltar as características dessas arenas conversacionais como espaços construídos e enfatizar as oportunidades para ação e os constrangimentos à interação social como fatores que influenciam crucialmente a conversação mediada e moldam as interações possíveis em cada situação.

À luz da teoria deliberativa e dos operadores frequentemente utilizados em pesquisas sobre deliberação online, propusemo-nos a pensar como alguns critérios, usualmente aplicados em estudos empíricos, podem contribuir para pensar conversações informais online - formas comunicativas e expressões políticas que foram ampliadas e enriquecidas com os novos dispositivos e recursos da Web 2.0.

Nosso objetivo foi realizar essa reflexão com base nas especificidades das trocas 
discursivas online, principalmente aquelas trazidas pelos próprios ambientes em suas características mais estruturais. Entendemos que o conteúdo, a forma, o processo discursivo que acontecem nesses espaços devem ser considerados para que se possa chegar a uma análise dos critérios sobre a qualidade das conversações políticas mais consonantes com a diversidade de plataformas e as interações estabelecidas hoje na internet. É preciso considerar, ainda, que os usuários encontram na internet possibilidades variadas de engajamento discursivo e, frequentemente, participam em mais de uma delas.

Contudo, ressaltamos que a nossa proposta apresenta algumas dificuldades. Primeiro, qualquer esforço para caracterizar a arquitetura tecnológica dos media não pode perder de vista que os sujeitos usam esses dispositivos para os mais diferentes propósitos. Tal uso é profundamente influenciado por um conjunto complexo de fatores socioculturais, que interagem com as oportunidades e os constrangimentos provocados pela interface de cada ambiente. Consequentemente, não há como fazer derivações neutras sobre as propriedades técnicas dos dispositivos, muito menos sugerir que eles sejam "positivos" ou "negativos" para a democracia.

Segundo, a indagação sobre a "conectividade" entre ambientes distintos de media, apesar de ter uma pretensão generalizante, pode apenas guiar estudos empíricos que precisam ser delimitados, a fim de construir certas peças de evidência, uma vez que as plataformas sociais da internet configuram um horizonte complexo de interações sociais que não pode ser considerado uniforme. Não obstante, entendemos que uma perspectiva mais nuançada sobre o ambiente digital pode ajudar os pesquisadores a interpretar os resultados de natureza micro, os quais são potencialmente válidos apenas em um dado contexto.

A abordagem aqui delineada valoriza o papel da conversação informal como processo catalisador de formas mais complexas de participação política e cívica. Entendemos, portanto, que a deliberação se dá em meio a práticas diversas e que ocorre em variadas instâncias, incluindo-se aí a arena dos media e os espaços de conversação informal (Mansbridge et al., 2012; Neblo, 2005; Maia, 2012; Steiner, 2012). No entanto, a abordagem da "ecologia complexa" do ambiente digital pode servir como ponto de partida para uma nova agenda de pesquisa. Nesse contexto, destacamos a investigação de ambientes cujos desenhos participativos criam oportunidades e constrangimentos distintos e, ainda, cujos espaços conversacionais são interdependentes. Essa perspectiva coloca o desafio de observar e investigar como os indivíduos se engajam em debates políticos em diferentes contextos, considerando a complexidade e a heterogeneidade de cada um deles. 
ROUSILEY C. M. MAIA ET AL.

Rousiley C. M. Maia - Professora associada do Departamento de Comunicação Social, Faculdade de Filosofia e Ciências Humanas, Universidade Federal de Minas Gerais (UFMG). E-mail: <rousiley@fafich.ufmg.br>.

Patrícia G. C. Rossini - Doutoranda em Comunicação Social na Faculdade de Filosofia e Ciências Humanas, Universidade Federal de Minas Gerais (UFMG) e pesquisadora do Grupo de Pesquisa em Mídia e Esfera Pública (EME/UFMG). E-mail: <patriciarossini@ufmg.br>.

Vanessa V. de Oliveira - Doutoranda em Comunicação Social na Faculdade de Filosofia e Ciências Humanas, Universidade Federal de Minas Gerais (UFMG) e pesquisadora do Grupo de Pesquisa em Mídia e Esfera Pública (EME/UFMG). E-mail: <vanessaveiga@ufmg.br>.

Alicianne G. de Oliveira - Doutoranda em Comunicação Social na Faculdade de Filosofia e Ciências Humanas, Universidade Federal de Minas Gerais (UFMG) e pesquisadora do Grupo de Pesquisa em Mídia e Esfera Pública (EME/UFMG). E-mail: <alicianneg@gmail.com>.

\section{Referências bibliográficas}

ACKLAND, R. Web social science: concepts, data and tools for social scientists in the digital age. Thousand Oaks, CA: Sage Publications, 2013.

Amaral, A.; Recuero, R.; Montardo, S. (orgs.). Blogs.com: estudos sobre blogs e comunicação. São Paulo: Momento Editorial, 2009.

BÄCHTIGER, A., et al. Measuring deliberation 2.0: standards, discourse types, and sequenzialization. In: ECPR General Conference, Potsdam, 2009. Disponível em:

<http://ash.harvard.edu/extension/ash/docs/baechtiger.pdf>. Acesso em: 13 fev. 2011.

BAYM, N. K. Personal connections in the digital age. Malden, MA: Polity Press, 2010.

BenhABIB, S. Toward a deliberative model of democratic legitimacy. In: BenHABIB, S. Democracy and difference: contesting the boundaries of the political. Princeton, NJ: Princeton University Press, p. 67-94, 1996.

BoHman, J. Public deliberation: pluralism, complexity and democracy. Cambridge, MA: MIT Press, 1996.

Bou-franCH, P.; LORENZO-DuS, N.; BLITVICH, P. G. "Social interaction in YouTube text-based Polylogues: a study of coherence". Journal of Computer-Mediated Communication, Bloomington, vol. 17, p. 501-521, 2012.

BOYD, D. "Social network sites: public, private, or what?". Knowledge Tree, Pittsburgh, 13 May 2007.

Social network sites as networked publics: affordances, dynamics, and implications. In: PAPACHARISSI, Z. (ed.). A networked self: identity, community and culture on social network sites. New York, NY: Routledge, p. 39-58, 2011.

BOYD, D; ElLISON, N. "Social network sites: definition, history and scholarship". Journal of Computer-Mediated Communication, Bloomington, vol. 13, n 1, p. 210-230, October 2007.

BRUNDIDGE, J. Political discussion and news use in the contemporary public sphere: the "accessibility" and "traversability" of the internet. In: Political Communication Division of The International Communication Association, Chicago, 2009. 
BRUndidge, J.; RICE, R. E. Political engagement and exposure to heterogeneous political discussion: do the (information) rich get richer and the similar get more similar? In: CHADWICK, A.; HOWARD, P. N. (eds.). The Handbook of Internet Politics. New York, NY: Routledge, p. 134-156, 2009.

Burgess, J.; Green, J. YouTube: online video and participatory culture. Cambridge, MA: Polity Press, 2009.

ChADWICK, A. "Web 2.0: New Challenges for the study of e-democracy in a era of informational exuberance". I/S: A Journal of Law and Policy, Columbus, vol. 5, n 1, p. 9-42, 2009.

. The hybrid media system: politics and power. Oxford, UK: Oxford University Press, $\overline{2} \overline{0} \overline{1} \overline{3}$. (Kindle edition).

CHADWICK, A.; MAY, C. "Interaction between states and citizens in the age of internet: 'egovernment' in the United States, Britain and the European Union". Governance: An International Journal of Policy, Administration and Institutions, Malden, vol. 16, n² 2, p. 271-300, April 2003.

COHEN, J. Deliberation and democratic legitimacy. In: BOHMAN, J.; ReHG, W. (eds.). Deliberative democracy. Londres, UK: MIT Press, p. 67-92, 1997.

COLEMAN, S.; BLUMLER, J. The internet and democratic citizenship: theory, practice and policy. Cambridge, MA: Cambridge University Press, 2009.

Coleman, S.; Moss, G. "Under construction: the field of online deliberation research". Journal of Technology \& Politics, New York, vol. 9, n 1, p. 1-15, 2012.

DAHLBERG, L. "The internet and democratic discourse: exploring the prospects of online deliberative forums extending the public sphere". Information, Communication \& Society, New York, vol. 4, $n^{\circ}$ 4, p. 615-633, 2001.

"Net-public sphere research: beyond the 'first phase'". Javnost - The Public, Liubliana, Eslovênia, vol. 11, n 1, p. 27-44, 2004.

"Rethinking the fragmentation on the cyberpublic: from consensus to contestation". New Media \& Society, Thousand Oaks, vol. 9, n 5, p. 5-26, 2007.

DAHLGREN, P. "The internet, public spheres and political communication: dispersion and deliberation". Political Communication, New York, vol. 22, p. 147-162, 2005.

Davies, T.; Chandler, R. Online deliberation design: choices, criteria, and evidence. In: NABATCHI, T., et al. (eds.). Democracy in motion: evaluating the practice and impact of deliberative civic engagement. Oxford: Oxford University Press, p. 103-131, 2012.

DONATH, J.; BOYD, D. "Public displays of connection". BT Technology Journal, New York, vol. 22, $\mathrm{n}^{\circ}$ 4, p. 71-82, 2004.

DRYZEK, J. S. Deliberative democracy and beyond: liberals, critics, contestations. Oxford, UK: Oxford University Press, 2000.

ElLISON, N. B.; BOYD, D. Sociality through social network sites. In: DutTON, W. H. (ed.). The Oxford Handbook of Internet Studies. Oxford, UK: Oxford University Press, p. 151-172, 2013.

Ellison, N. B.; Steinfield, C.; LAMPE, C. "Social capital implications of Facebook-enabled: communication practices". New Media Society, Thousand Oaks, vol. 13, n 6, p. 873-892, 2011. 
ROUSILEY C. M. MAIA ET AL.

ELLISON, N., et al. With a little help from my friends: how social network sites affect social capital processes. In: PAPACHARISSI, Z. (ed.). A networked self: identity, community, and culture on social network sites. Cambridge, MA: Polity Press, p. 124-145, 2010.

GrAHAM, T. "Needles in a haystack: a new approach for identifying and assessing political talk in nonpolitical discussion forums". Javnost - The Public, Liubliana, Eslovênia, vol. 15, n² 2, p. 17-36, 2008.

Grasmuck, S.; MARTIN, J.; ZHAO, S. "Ethno-racial identity displays on Facebook". Journal of Computer-Mediated Communication, Bloomington, vol. 15, n 1, p. 158-188, 2009.

Gutmann, A.; Thompson, D. Democracy and disagreement. Cambridge, MA: Harvard University Press, 1996.

Gutmann, A.; ThOMPSON, D. (eds.). Why deliberative democracy? Princeton, NJ: Princeton University Press, 2004.

HABERmAS, J. Direito e democracia: entre facticidade e validade. Rio de Janeiro: Tempo Brasileiro, 1997.

JANSSEN, D.; KIES, R. "Online forums and deliberative democracy: hypotheses, variables and methodologies". Conference Empirical approaches to deliberative politics. EUI, Florence, 2004.

JENKInS, H. Convergence culture. New York, NY: NYU Press, 2006.

Cultura da convergência. Trad. Susana Alexandria. 2ª̣ ed. São Paulo: Aleph, 2009.

JENKINS, H., et al. Spreadable media: creating value and meaning in a networked culture. New York, NY: New York University Press, 2012.

JURIS, J. "The new digital media and activist networking within anti-corporate globalization movements". The Annals of the American Academy of Political and Social Science, Philadelphia, vol. 597, p. 189-208, 2005.

KAYE, B. Between Barack and a net place: users and uses of social network sites and blogs for political information. In: PAPACHARISSI, Z. (ed.). A networked self: identity, community and culture on social network sites. New York, NY: Routledge, p. 208-231, 2011.

KIES, R. Promises and limits of web-deliberation. Nova York, NY: Palgrave Mcmillan, 2010.

LINDGREN, S. "'It took me half an hour, but I did it!'. Media circuits and affinity spaces around howto videos on YouTube". European Journal of Communication, Thousand Oaks, vol. 27, $\mathrm{n}^{\circ} 2$, p. 152$170,2012$.

MAIA, R. C. M. Deliberation, the media and political talk. Nova York: Hampton Press, 2012.

Struggles for recognition in the digital era. In: MAIA, R. C. M. Recognition and the media. Hampshire/Nova York, p. 103-122, 2014.

MAIA, R. C. M.; REZENDE, T. A. S. Recognition as ongoing struggle: conflicts involving racism and homofobia in the networked media environment. In: MAIA, R. C. M. Recognition and the media. Hamphshire/Nova York, p. 123-147, 2014 a.

Deliberative system and discussions on racism and homophobia in networked media environment. In: Annual Conference International Communication Association, 64th, Seattle, 2014b. 
MANIN, B. "On legitimacy and political deliberation". Political Theory, Thousand Oaks, vol. 15, $\mathrm{n}^{\circ} 3$, p. 338-368, August 1987.

MANSBRIDGE, J. Everyday talk in the deliberative system. In: MACEDO, S. Deliberative politics: essays on democracy and disagreement. New York, NY: Oxford University Press, p. 211-239, 1999.

MANSBRIDGE, J., et al. A systemic approach to deliberative democracy. In: PARKINSON, J.; MANSBRIDGE, J. Deliberative systems: deliberative democracy at the large scale. Cambridge, MA: Cambridge University Press, p. 1-26, 2012.

MARQUES, A. C. S.; MAIA; R. C. M. "Everyday conversation in the deliberative process: an analysis of communicative exchanges in discussion groups and their contributions to civic and political socialization". J ournal of Communication, Hoboken, vol. 60, p. 611-635, 2010.

MerAZ, S. Analyzing political conversation on the Howard Dean candidate blog. In: TREMAYNE, M. (ed.). Blogging, citizenship, and the future of media. New York: Routledge, p. 59-82, 2007.

MUTZ, D. Hearing the other side: deliberative versus participatory democracy. Cambridge, MA: Cambridge University Press, 2006.

NEBLo, M. "Thinking through democracy: between the theory and practice of deliberative politics". Acta Politica, Brunel Road, vol. 40, p. 169-181, 2005.

NORRIS, P. A virtous circle: political communications in postindustrial societies. Cambridge, MA: Cambridge University Press, 2000.

O'ReILLY, T. What is Web 2.0 - design patterns and business models for the next generation of software. Sebastopol, CA: O'Reilly Publishing, 2005.

PAPACHARISSI, Z. "Democracy online: civility, politeness, and the democratic potential of online political discussion groups". New Media and Society, Thousand Oaks, vol. 6, n 2, p. 259-283, 2004.

. A private sphere. Democracy in a digital age: digital media and society series.

Cambridge, MA: Polity Press, 2010.

RISSE, T. "Let's argue! Communicative action in international relations". International Organization, Cambridge, vol. 54, p. 1-39, 2000.

Rossinı, P. G. C. "O uso de redes sociais e comportamento eleitoral: a influência do Facebook na decisão do voto nas eleições 2012 em Juiz de Fora". Dissertação de Mestrado em Comunicação. Programa de Pós-Graduação em Comunicação, Universidade Federal de Juiz de Fora, 2013.

RossinI, P. G. C.; MAIA, R. C. M. Is political participation online effective? A case study of the edemocracy project conducted by the Federal Chamber of Deputies in Brazil (pre-print). In: SONNTAGBAUER, P., et al. (eds.). Advanced ICT integration for governance and policy modeling: sharing policy decisions in the digital world. Viena: IGI Global, p. 341-362, 2014.

SAMPAIO, R. C.; BARRos, S. A. R.; MoraIS, R. "Como avaliar a deliberação online?: um mapeamento de critérios relevantes". Opinião Pública, Campinas, vol. 18, p. 470-489, 2012.

SHIRKY, C. Cultura da participação: criatividade e generosidade no mundo conectado. Rio de Janeiro: Zahar, 2011.

SILES, I. "The rise of blogging: articulation as a dynamics of technological stabilization". New Media \& Society, Thousand Oaks, vol. 14, n 5, p. 781-797, 2012. 
ROUSILEY C. M. MAIA ET AL.

STEINER, J. The foundations of deliberative democracy. Empirical research and normative implications. Cambridge, MA: Cambridge University Press, 2012.

STEINER, J., et al. Deliberative politics in action: analysing parliamentary discourse. Cambridge, MA: Cambridge University Press, 2004.

StRomer-GALEY, J. "Measuring deliberation's content: a coding scheme". Journal of Public Deliberation, Louisville, vol. 3, n 1, p. 1-35, 2007.

Valenzuela, S.; PARK, N.; KeE, K. F. "Is there social capital in a social network site?: Facebook use and college students' life satisfaction, trust, and participation". Journal of Computer-Mediated Communication, Bloomington, vol. 14, $\mathrm{n}^{\circ} 4$, p. 875-901, 2009.

. "The role of friends behaviour on evaluations of individuals Facebook profiles: are we known by the company we keep?". Human Communication Research, Hoboken, vol. 34, p. 28-49, 2008.

WALTHER, J., et al. Interaction of interpersonal, peer, and media influence sources online: a research agenda for technology convergence. In: PAPACHARISSI, Z. (ed.). A networked self: identity, community and culture on social network sites. New York, NY: Routledge, p. 17-38, 2011.

WARD, J. Social media and democracy: innovations in participatory politics. New York, NY: Routledge, 2012.

WARREN, M. Institutionalizing deliberative democracy. In: RosenBERG, S. W. (ed.). Can the people govern? Deliberation, participation and democracy. New York, NY: Palgrave Macmillan, p. 272-310, 2007.

WilheLM, A. Democracy in the digital age: challenges to political life in cyberspace. New York, NY: Routledge, 2000.

WoJCIESZAK, M.; MUTZ, D. "Online groups and political discourse: do online discussion spaces facilitate exposure to political disagreement?". Journal of Communication, Hoboken, vol. 59, $n^{\circ} 1$, p. 40-56, March 2009.

WRIGHT, S.; StREET, J. "Democracy, deliberation and design: the case of online discussion forums". New Media \& Society, Thousand Oaks, vol. 9, n5 p. 849-869, 2007.

\section{Resumo}

Sobre a importância de examinar diferentes ambientes online em estudos de deliberação

Diversos autores conceituam a Web 2.0 como uma "ecologia complexa" a fim de apreender as novas configurações da esfera pública nas sociedades contemporâneas. Contudo, raros estudos desenvolvem uma caracterização sistemática da especificidade das condições de comunicação nos ambientes digitais. Este artigo tem por objetivo caracterizar, numa base comparativa, três espaços de conversação online: blogs, sites de redes sociais (como o Facebook) e plataformas de conteúdo colaborativo (como o YouTube). A partir de uma exploração crítica da literatura corrente e de estudos empíricos, examinam-se as implicações da identificação dos participantes, o papel da moderação, a interatividade social e a natureza do público presumido. Tomando como pano de fundo as condições normativas para a deliberação, defende-se o argumento segundo o qual a relação entre o design e a organização dos espaços virtuais tem impactos específicos na 
configuração da argumentação e da conversação mediada. Como conclusão, indicamos algumas implicações da arquitetura técnica de distintas plataformas para pensar o ambiente complexo das conversações online sob uma perspectiva deliberacionista.

Palavras-chave: deliberação; conversação; mídias sociais; YouTube; Facebook; blogs

\begin{abstract}
About the importance of analyzing different online environments in deliberation studies

Several scholars have proposed the conceptualization of the Web 2.0 as a "complex ecology" in order to grasp the new settings of the public sphere in contemporary societies. However, few studies have developed an accurate characterization of the specific conditions of communication on digital environments. This article aims to characterize, on a comparative basis, three conversation online spaces: blogs, social network sites (i.e. Facebook) and collaborative platforms (i.e. YouTube). From a critical assessment of the current literature and empirical studies, it focuses on the implications of the identification of participants, the role of moderation, the social interactivity and the nature of the expected public. Taking into consideration the normative conditions for deliberation, we defend the argument that the relationship between the platform design and the organization of conversational online spaces has specific impacts on mediated conversation and argumentation setting. In conclusion, we indicate some implications of technical architecture of distinct platforms in order to think about the complexity of online conversations under a deliberative perspective.
\end{abstract}

Keywords: deliberation; conversation; social media; YouTube; Facebook; blogs

Artigo submetido à publicação em agosto de 2014. Aprovado para publicação em fevereiro de 2015. 